\title{
Besprechungen.
}

I .

E. Lexer, Wiederherstellungschirurgic. I. Abschnitt. Beseitigung von Gewebsdefekten. 235 Abb., I62 S. Preis brosch. M. 12. Leipzig, J. A. Barth, I919.

In verschiedenen Kapitcln ist die Bescitigung. von Knochen-, Gelenk-, Muskel-, Nerven- und Gefäßdefekten behandelt, wobei den Knochendefekten der breiteste Raum gewidmet ist. Es ist nicht die Absicht des Autors, wie im Vorwort auch eigens hervorgehoben wird, eine Zusammenstellung aller für die Wiederherstellung dieser Defektc gangbaren Verfahren zu geben. Die Arbeit stellt vielmehr einen Gesamtbericht des Meisters einer der hervorragendsten deutschen Werkstätten der Wiederherstellungschirurgie dar, die als Geburtsstïttc so mancher in das Allgemeingut der Chirurgie ubergegangenen Verfahren hinlänglich bekannt ist. Es ist die Zusammenfassung von Lexers persönlicher Erfahrung in der Wiederherstellung der Kriegsschäden. In dieser persönlichen Darstellung liegt gerade der Wert der Monographie, die sich auch deshalb nicht zu einem trockenen Refcrate eignet. Die angewandten Verfahren sind dem Kundigen zum größten Teil nicht mehr neu, aber er bekommt hier wertvolle Winke für die Art, wie die Verfahren angewendet werden sollen. Die Angaben zum Beispiel über die Indikation zur Pseudarthrosenoperation, über die Wahl des Zeitpunktes, über die Berücksichtigung der ruhenden Infektion, die Art der Anwendung der Autoplastik, namentlich der Anpassung der 'Transplantate, über Schädelplastik, über den Ersatz von Kieferdefekten und Kieferaufbau und vielem anderen wirken in dieser Richtung hin ungemein eindrucksvoll und lassen allein die Monographie jedem Chirurgen zum Studium empfehlen, der sich mit diesen Dingen beschäftigen will. Auffallend sind die günstigen Erfolge, die L exer entgegen den Erfahrungen manch anderer Autoren immer wieder mit Homoiplastik zu verzeichnen hat. - Sanitätsrat L. L u d w ig hat die Darstellung durch ein anschaulich greschriebenes Kapitel über die zahnärztliche Behandlung der Kieferverletzungın ergänzt. - Zahlreiche gute Abbildungen illustrieren den Text, zahlreiche ausführliche Krankengeschichten bildet die dokumentarische Basis der niedergelegten Erfahrungen.

E. v. Redwitz (Heidelberg). 
2.

F Thedering (Oldenburg), Das $Q u a r z l i c h t$ und seine Anwendung in der Medizin. 3. Auflage. Verlag G. Stalling, Oldenburg. 15.9 Seiten, I5 Abbildungen. Preis M. 9.-.

Das Buch liegt in kurzer Zeit nunmehr in der 3. Auflage vor, ein Beweis für das Bedürfnis eines solchen und die Zweckmäßigkeit desselben. Die neue Auflage weist verschiedene Verbesserungen und Erweiterungen auf. Namentlich ist es ein Kapitel über Rotlichtbehandlung und Licht und Farbe als Energieträger, das neu hinzugekommen ist, andere, wie das über die biologische Lichtwirkung, haben Erweiterung erfahren. Die große Bedeutung des Quarzlichtes für die Wundbehandlung ist in einem besonderen Kapitel gewürdigt, womit dasjenige über das Quarzlicht im Dienste des Kriegslazarettes fortfallen konnte. Es folgen dann wiederum wie bisher die verschiedenen Hauterkrankungen und ihre Behandlung mit der Quarzlampe und zum Schluß noch einige allgemeinere Anwendungsformen.

Das Literaturverzeichnis ist auf 449 Nummern angewachsen. B a i s ch (Heidelberg).

3.

E. Sommer (Zürich), Röntgentaschenbuch. VIII. Bd. 1919. Verlag von Otto Nemnich, München-Leipzig. 288 Seiten mit 6i Abbildungen u. 4 Tafeln. Preis M. 8.-.

Das bekannte Röntgentaschenbuch liegt im 8 . Band vor und bringt wieder wie bisher einen technisch-diagnostischen Teil, einen therapeutischen Teil und einen Teil, der die Übersicht über Leistungen und Fortschritte der röntgenologischen Technik 1916/17 gibt. In den ersten beiden, klinischen Abschnitten sind eine Anzahl (23) kurze Abhandlungen, die neuere Probleme der Diagnostik und Therapie behandeln und dadurch leicht und rasch über die wesentlichen Fortschritte auf dem Gebiet orientieren. Im therapeutischen Abschnitt ist es namentlich die Schwerfilterbehandlung, die von verschiedenen Seiten empfohlen wird. Der technische Teil bringt Mitteilungen aus der Röntgenindustrie über Neuerungen und Verbesserungen an Apparaten und Röhren, wobei die Glühkathodenröhre und die verschiedenen Formen der Siederohre eine große Rolle spielen.

$\mathrm{B}$ a is ch (Heidelberg).

4.

Die Lichtbehandlung (Heliotherapie in den deut. schen Lungenheilanstalten. - Denkschrift bearbeitet v. San.-Rat Dr. G. Li ebe mit Beiträgen von Prof. Dr. Bacmeister, Dr. Bochalli und Dr. Scheffer. 
6I Seiten, 2 Abbildungen. Verlag Curt Kabitzsch. Leipzig I9I9. Preis M. 3.50.

Die Denkschrift baut sich auf einem durch Rundfragen bei den deutschen Lungenheilstätten gewonnenen Material auf. Von 85 Anstalten hatten 55 geantwortet. Es werden Luft- und Sonnenbad, künstliches Lichtbad, Röntgentiefentherapie und Glühlichtbad besprochen; ein längerer Abschnitt über die Theorie der'Lichwirkungen folgt der Besprechung von Sonnen- und künstlichen Lichtbädern. Über Röntgentiefentherapie bei Lungentuberkulose und deren Verbindung mit Lichttherapie gibt B a cmeister auf sieben Seiten eine außerordentlich klare Darstellung. Nach ihm wirken die Röntgenstrahlen, wie überall auch sonst im Körper, auf junges, rasch wachsendes, hier also auf das Granulationsgewebe. Dies wird zerstört und durch Narbengewebe ersetzt. Es eignen sich darnach für Röntgentiefentherapie „die Fälle der zur Latenz neigenden, der stationären und der langsam progredienten Formen der indurierenden und desiminierten Lungentuberkulose", kontraindiziert ist die Strahlenanwendung bei pneumonisch-exsudativen, destruierend-kavernösen und akutdestruierenden Formen. Vieles, vor allem die Darstellung über die Theorie der Strahlenwirkungen, gilt wohl ebenso wie für die Lungenauch für die chirurgischen Tuberkulosen. Dieser Umstand erhöht für den Chirurgen noch den Wert der Arbeit, der ihr auch bei engerer Fassung des Themas vermöge der Wichtigkeit des Gegenstandes zukäme. Reichliche Literaturnachweise machen die Denkschrift auch zu einem brđuchbaren Nachschlagewerk.

v. B o $\mathrm{n}$ in (Heidelberg).

\section{5 .}

Primäre Excision und primäre Naht akzidenteller Wunden. - Von Prof Dr. Robert Bárány (Upsala). 256 Seiten. Leipzig und Wien, Franz Deuticke I919. Preis M. 15.-, Kr. 18.-

Bá rá n y beginnt sein Werk mit einem geschichtlichen Überblick über die Lehre der Wundbehandlung, fängt mit Hippocrates und Celsus an, führt den Leser dann über die Antisepsis und Asepsis, über Lister, Semmelweis, v. Bergmann und andere Autoren zu dem Hauptthema des Buches, zur primären Naht der Schußwunden.

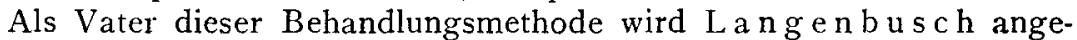
sehen, welcher eine Reihe von Gegnern fand. Sodann werden die Arbeiten von P. L. Friedrich besonders gewürdigt, welcher als erster die Excision der Wundränder empfohlen hat und so versuchte eine Wundheilung mit prima intentio $z u$ erzielen. Es folgt dann eine historische Übersicht über die Behandlung der Schädel- und Hirnwunden und darauf die Schilderung der Kriegschirurgie vor Beginn des Krieges 1914, besonders in bezug auf die Kopfverletzungen. 
Verfasser kommt dann auf seine eigenen Erfahrungen im Kriegre zu sprechen, schildert sehr ausführlich seinen Wirkungskreis und seine kriegsärztliche Tätigkeit und zitiert seine bisherigen A.rbeiten über Schußverletzungen des Schädels und des Gehirns, teilt uns sogar mit, daß zwei dieser Arbeiten durch Fliegerpost (!) von Przemysl weggeschickt worden sind. Er bespricht dann des Genaueren seine Erfahrungen über $\mathrm{Hirnabszesse}$ an der Hand von I2 Fällen, die ja demjenigen, welcher während des Krieges Gelegenheit hatte, sich mit diesen Dingen zu beschäftigen, nichts wesentlich Neues bringen.

Es folgen dann an der Hand der schon früher publizierten I2 geheilten Fälle dic Ansichten des Verfassers über die primäre Naht der $f r i s c h e n$ Gehirnschüsse, eine Methode, für die ja, wie bekannt, Bárány sehr warm eintritt, ebenso wie für die primäre Excision und Naht der übrigen Schußwunden. Diese Ansichten des Verfassers bilden den Kernpunkt des ganzen Werkes. Der übrige, größte Teil des Buches bespricht in sehr breiten Aus. führungen die Methoden und Erfolge anderer Autoren des Inund Auslandes hebt die Arbeiten derjenigen Operateure hervor, die nach gleicher oder ähnlicher Methode gearbeitet haben wie der Verfasser, stellt aber im wesentlichen eine Polemik dar gegen alle Kriegschirurgen, welche die primäre Naht der Hirnwunden, zum Teil nach sehr reichlichen Erfahrungen, $\mathrm{nicht}$ für angezeigt halten. Die Zahl dieser Autoren ist eine sehr große.

$$
\text { M a nasse (Würzburg). }
$$

\section{6.}

Steinmann, Dr. Fritz, Lehrbuch der funktionellen Behandlung der Knochenbrüche und Gelenkverletzungen' für Ärzte und Studierende. Mit 270 Textabbildungen. Verlag von Ferd. Enke, Stuttgart I9I9. Preis M. I8.-.

Das Steinmannsche Buch ist kein systematisches Lehrbuch der Frakturenlbehandlung, sondern beschäftigt sich fast aus. schließlich mit der frühzeitigen $B$ ewegungsitherapie bei Knochenbrüchen. Insofern eignet es sich weniger für den lernenden Studenten, als für den Arzt, besonders für den Chirurgen. Seine Lektüre erfordert ein kritisches Urteil, dann ist sie aber auch außerordentlich genußreich und nutzbringend. - Diese rein subjektive Darstellung der Frakturbehandlung macht es selbstverständlich, daß man in vielen Punkten anderer Meinung sein kann, als der Verfasser. Daneben bringt aber das Buch sehr viele beachtenswerte, aber leider noch nicht genügend beachtete Ratschläge für die Behandlung von Knochenbrüchen, und es kann deshalb seine Lektüre jedem Chirurgen nur angelegentlich empfohlen werden. - Wenn sich die künftigen 
Auflagen an einen weiteren Kreis von Lesern wenden sollen, so wäre eme etwas ausführlichere Darstellung der allgemeinen Frakturenlehre wünschenswert. Die Zuppingerschen Lehren sind doch eben noch nicht so Allgemeingut geworden, wie in dem Steinmannschen Buch vorausgesetzt wird. Auch sollten die Unterschiede in der Behandlung der Knochenbrüche bei Kindern und Erwachsenen mehr betont werden, selbst wenn dann der Verfasser bei der kritischen Durchsicht seiner Fälle (z. B. suprakondyläre Fraktur) zu der Ansicht kommen müßte, daß die Einrichtung eines Knochenbruches und eine Fixation in der Gips-Hanfschiene für manche Fälle von kindlichen Knochenbrüchen doch noch das beste Verfahren ist und jedenfalls weniger gefährlich als die empfohlene blutige Freilegung und Nagelung.

$R$ ost (Heidelberg).

\section{7.}

Tandler, Julius, Lehrbuch der systematischen Anato mi e. I. Band, 3. Heft. Myologie. Verlag: F. C. W. Vo. gel, Leipzig 1919. Preis M. 6.-.

Mit dem vorliegenden 3 . Hefte ist der I. Band von Tandlers Anatomie abgeschlossen. Er enthält die Darstellung der Muskulatur in einer für den Studierenden, Arzt und Chirurgen ungemein übersichtlichen und praktisch sehr brauchbaren Weise. Der I. Teil behandelt die allgemeine Muskellehre, der 2. die spezielle. In der Anordnung der einzelnen Muskeln folgte der Autor nicht nur dem topographischen Prinzipe, sondern berücksichtigte auch das entwicklungsgeschichtliche Moment. Sehr zu begrüßen ist auch der Umstand, daß bei jedem Muskel auch die Innervation, die segmentale Zugehörigkeit und die Wirksamkeit angegeben ist. Sehr zweckmäßig werden bei den Muskeln auch die Fascien abgehandelt. Chirurgisch wichtige Partien, wie z. B. der Leistenkanal und Schenkelkanal, erklärt der Autor besonders sorgfältig, ohne sich in unwichtige Einzelheiten einzulassen. Überhaupt kann man auch bei der Muskellehre wie bei der Knochen- und Gelenkslehre hervorheben, da $B$ sie keinen unnötigen Ballast bringt, das Wissenswerte sehr einfach und klar darstellt, aber dabei auch anscheinend geringfügige Verhältnisse betont, wenn sie von praktischer Bedeutung sind. Die farbigen Abbildungen machen einen vorzüglichen Eindruck und gehören wohl zu den besten auf diesem Gebiete. Mögen bald die beiden übrigen Bände von Tandlers Anatomie erscheinen.

$$
\mathrm{N} \text { a r a th (Heidelberg). }
$$

